The term 'septal pore' proposed by Hashimoto et al. ${ }^{4}$ is not accepted in this case since this term has been applied to the much larger pores of the ascomycete and basidiomycete type. The term 'septal micropore' is proposed for the pore type seen in Geotrichum candidum.

Hashimoto et al. ${ }^{4}$ have likened the small pores observed to the pits in higher plant cell walls. This interpretation should not be accepted because the pit of a higher plant is an unthickened part of a secondary wall, and plasmodesmata in higher plants are the cytoplasmic connexions between adjacent cells. In the ascomycete and basidiomycete type of pore the intercellular connexion is not considered a plasmodesmata by virtue of the way in which they are formed ${ }^{3}$. The 'septal micropores' shown here, however, are of the nature of plasmodesmata.

We thank Dr. H. J. Swart for supplying the culture of the fungus studied.

Department of Botany, University of the Witwatersrand, Johannesburg, South Africa.

${ }^{3}$ Shatkin, A. J., and Tatum, E. L., J. Biophys. Biochem. Cytol., 6, 423 (1959). Moore, R. T., and McAtear, J. H., Amer. J. Bot., 49, 86 (1962).

- Bracker, jun., C. E., and Butler, E. E.. Mycologia, 55, 35 (1963).

- Hashimoto, T., Kishi, T., and Yoshida, W., Nature, 202, 1353 (1964),

s Luft, J. H., J. Biophys. Biochem. Cytol., 9, 409 (1961).

- Feldman, D. G., J. Cell Biol., 15, 592 (1962).

\section{Retarded Embryo Sac Development in Irradiated Clones of Malus silvestris Hort. cv. 'Golden Delicious'}

Seven 'Golden Delicious' apple clones propagated from buds irradiated at $5,000 \mathrm{r}$. in a cobalt- 60 source were selected for high, intermediate, and low pollen abortion. Pollen abortion was 4.9 per cent in a typical check clone, and varied in the irradiated clones from 10.4 per cent to 84.8 per cent. Blossoms collected at anthesis were sectioned serially at $10 \mu$ thickness and stained with Heidenhain's iron alum haematoxylin for embryo sac examinations.

Increased pollen abortion was associated with decreased proportions of differentiated (migrating polar nuclei) 8-nucleate embryo sacs. The check clone had 52.2 per cent of its embryo sacs at this stage; the treated clones with 52.9 per cent or less pollen abortion (group 1) had $36 \cdot 2$ per cent at this stage; the intermediate clone (group 2) with 68.0 per cent aborted pollen had $24 \cdot 3$ per cent of its embryo sacs at this stage; and the clones with $70 \cdot 6$ per cent or more pollen abortion (group 3) contained only 9.6 per cent differentiated 8-nucleate embryo sacs. There was a highly significant negative rank correlation $\left(r_{s}=\right.$ -0.952 ) between the percentage pollen abortion and the percentage of differentiated 8-nucleate embryo sacs.

As pollen abortion increased, relatively more embryo sacs preceded an 8-nucleate stage. The check clone had
$14 \cdot 6$ per cent of its embryo sacs at the 2- or 4-nucleate stage; group 1 had 20.5 per cent; group 2 had 31.4 per cent; and group 3 had 36.4 per cent of its embryo sacs at this stage. Megaspore mother cells and megaspores were found only in clones with 70.6 per cent or more pollen abortion, except that one megaspore was found in clone 23-26. There was a highly significant positive correlation $\left(r_{s}=0.952\right)$ between pollen abortion and the total percentage of megaspore mother cells, megaspores, and 2 or 4-nucleate embryo sacs. The average number of ovules per pistil was fairly uniform from clone to clone and showed no clear relationship to pollen abortion (Table 1). Irradiation did not materially reduce the proportion of normally developing embryo sacs, or influence the relatively infrequent occurrence of supernumerary or degenerate embryo sacs.

The results of investigations with this cultivar suggest that irradiation resulted in retardation of female gametophyte development. Furthermore, there was a close association between pollen abortion and retarded embryo sac development.

We thank K. Lapins of the Canada Department of Agriculture, Research Station, Summerland, British Columbia, for his advice and for making available his irradiated clones for blossom collections. This work was supported by the National Research Council of Canada.

\section{B. H. Barritt}

G. W. EATON

Division of Plant Science, University of British Columbia,
Vancouver 8, B.C., Canada.

\section{Effect of Light Intensity on the Metabolic Path- ways in Photosynthesis}

TraCER experiments indicate that different substances (carbohydrates, proteins, lipids) are synthesized in plants, even after a short exposure to ${ }^{14} \mathrm{CO}_{2}$ (ref. 1). Therefore, although photosynthesis starts with a single primary reaction, a number of photosynthetic pathways lead to various photosynthetic products. We have investigated the effect of light intensity on these alternative pathways.

The investigations were made in a light thermostat ${ }^{2}$, using Phaseoluts vulgaris L. (var. 'Sugar Bean') plants grown in sand culture. Low light intensities were applied because the effect of the light factor (near the minimum) is in this way more pronounced. Carbohydrates ${ }^{3}$ and nitrogen ${ }^{4}$ were estimated from three-week-old plants.

It was found that an increasing light intensity proportionally raises the relative carbohydrate contents of the plants (Fig. 1). This rise seemed to be steady in the starch and soluble carbohydrate fractions up to 2,000 lux; however, at 4,000 lux there was a dramatic increase in the starch fraction of the leaf and in the soluble carbohydrate fraction of the stem.

Table 1. Condition of Embryo Sacs in Check aNd IrRadiated 'Golden Delicious' Apple Clones arranged in ORder of INCREasing Pollen Abortion

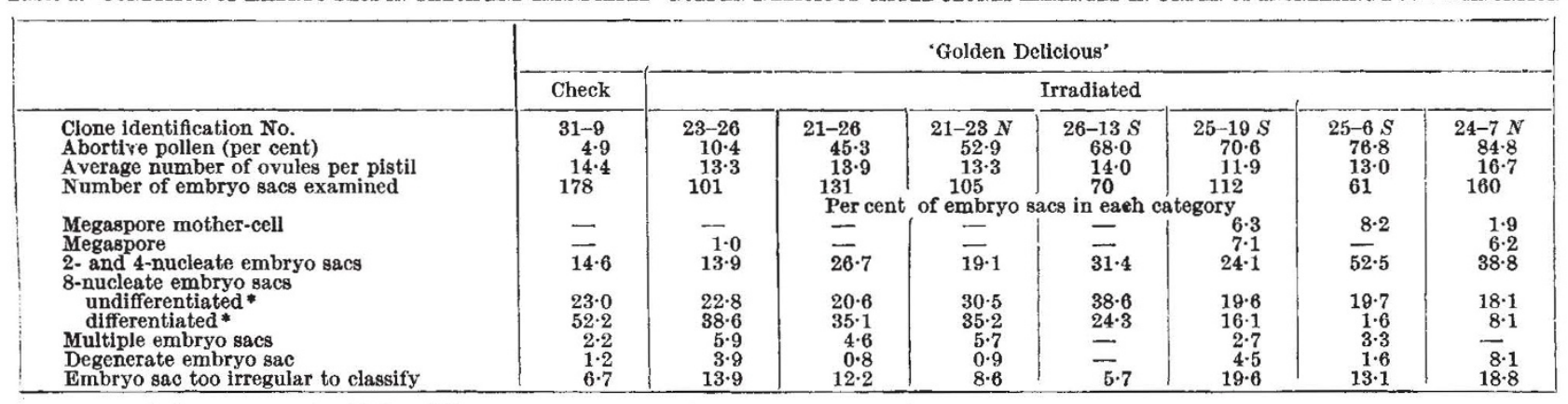

* 8-nucleate sacs were classifled as differentiated when the polar nuclei had begun to migrate. 